\title{
Measurement of the Electron Beam Point Spread Function (PSF) in a Scanning Electron Microscope (SEM)
}

\author{
Yudhishthir P. Kandel ${ }^{1}$, Matthew D. Zotta ${ }^{1}$, Andrew N. Caferra ${ }^{1}$, Richard Moore ${ }^{2}$, and Eric Lifshin ${ }^{1}$
}

${ }^{1}$ College of Nanoscale Science and Engineering, SUNY Polytechnic Institute, Albany, NY, USA

${ }^{2}$ Nanojehm Inc. Albany, NY, USA

A knowledge of the spatial distribution of the electron beam current density, often referred to as the point spread function (PSF), is valuable for understanding the behavior of scanning electron microscopes (SEM) and various other instruments. Previously, a number of attempts at PSF determination have been made based on experimental measurements or electron optical calculations [1-7]. Some of the experimental methods employed knife edge or other scans[1, 8]. Liddle et al. [5] used a TEM image of a reference sample to determine the PSF for an electron beam lithography tool. They assumed an elliptical Gaussian shape for the electron beam and determined its standard deviations in two orthogonal directions using an iterative method to match the reference and blurred images. Babbin et al. developed a test sample that can be used to estimate the PSF using a Fourier transform method[6]. All these approaches are limited in the sense that they do not provide the fine, often irregular, details in the electron beam shape that may not be symmetric or monotonic. A more accurate determination of a PSF is critical, however, if the goal is to improve SEM resolution by deconvolution using the method described by Lifshin et. al [9].

PSF determination described here is based on the availability of a well characterized near planar reference sample with a very small secondary electron mean free path. The intensity map of the reference sample is designated by the matrix X. It is measured under conditions such that the probe size is comparable to the pixel size. Furthermore, a high enough probe current and data collection time is selected to ensure an adequate signal to noise ratio. The reference sample is then imaged with either the same or a different microscope using different operating conditions to get image $b$, where the probe size may be considerably larger than the pixel size. These imaging conditions typically correspond to practical operating conditions such as the use of large probe currents needed to obtain low noise images in a short time as is the case for a thermionic source SEM. If the PSF, K, is assumed to be position independent, which we have verified experimentally for a range of conditions, then the problem of finding electron beam shape can be posed with the use of, $\Gamma$, the regularization functional.

$$
\begin{array}{ll}
\underset{\text { w.r.t. } K}{\operatorname{minimize}} & f(K \otimes X-b)+\Gamma(X, b ; \lambda) \\
\text { subject to } & K \succeq 0 ; \quad\{f, \Gamma\}: \mathbb{R}^{m \times n} \rightarrow \mathbb{R}
\end{array}
$$

As a prerequisite for implementation of this method, procedures have been developed for proper sub-pixel image alignment and the elimination of artifacts present from digitization, brightness, contrast, and gamma settings as well as saturation effects.

As an example, Figure 1 shows a three dimensional PSF near the electron beam focus measured with a TESCAN VEGA ${ }^{\circledR}$ LaB $_{6}$ source SEM operated at $20 \mathrm{keV}$ and $7 \mathrm{pA}$ of probe current. SEM images of an $\mathrm{Au}-\mathrm{C}$ Pella ${ }^{\circledR}$ sample corresponding to various focus positions and the reference image are also shown. Since the roughness of the sample was less than one micrometer and the PSF did not change significantly over the first one micrometer step shown, the value around the zero displacement setting was found to 
successfully lead to image restorations with improved spatial resolution. Research is underway to use the full PSF in dealing with structures with a higher degree of roughness.

The authors acknowledge the support of Mr. Jeffrey Moskin, President of Nanojehm for providing the resources that made this study possible as well as TESCAN for providing instrumental support.

\section{References:}

1. E. Kratschmer, S. A. Rishton, D. P. Kern and T. H. P. Chang, JVST B 6 (6), 2074-2079 (1988).

2. M. Sato, JVST B: Microelectronics and Nanometer Structures 9 (5), 2602 (1991).

3. J. E. Barth and P. Kruit, optik 109 (3), 101-109 (1996).

4. A. Goldenshtein, Y. I. Gold and H. Chayet, SPIE 3332, 132-137 (1998).

5. $\quad$ J. A. Liddle, P. Naulleau and G. Schmid, JVST B 22 (6), 2897 (2004).

6. S. Babin, M. Gaevski, D. Joy, M. Machin and A. Martynov, JVST B 24 (6), 2956-2959 (2006).

7. K. Nakamae, M. Chikahisa and H. Fujioka, Image and Vision Computing 25 (7), 1117-1123 (2007).

8. T. A. P. J.W. Elmer, A.T. Teruya, presented at the 2nd International Conference on Electron Beam Welding, Aachen, Germany, 2012 (unpublished).

9. $\quad$ E. Lifshin, Y. P. Kandel and R. L. Moore, Microscopy and Microanalysis 20 (01), 78-89 (2014).

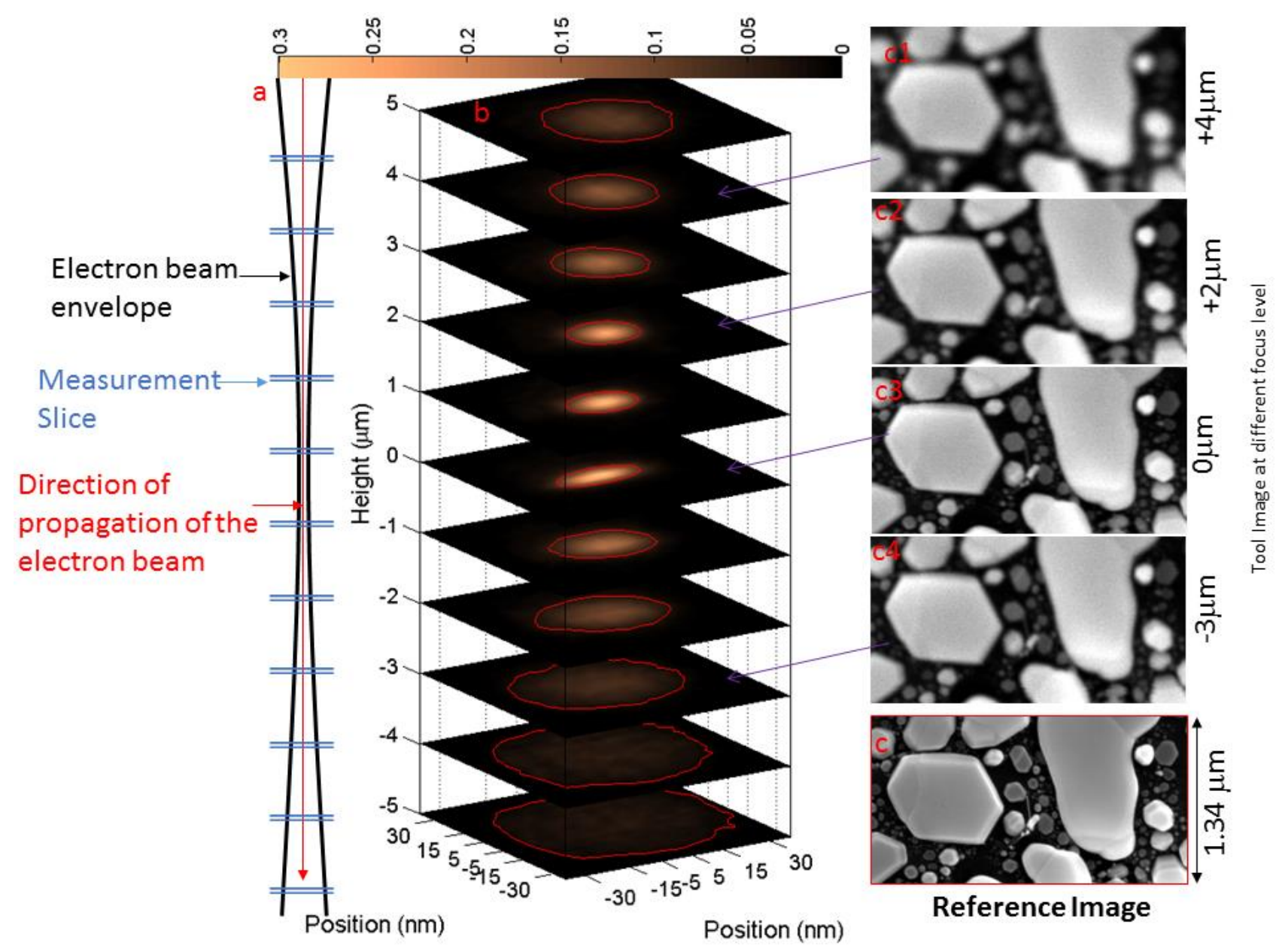

Figure 1: (a) shows the general electron beam envelope near focus, result from measurement slices taken at various locations as measured in TESCAN VEGA is shown in (b) as a three dimensional color plot; (c) is the reference image taken with TESCAN MIRA ${ }^{\circledR}$ (c1), (c2), (c3), and (c4) shows the images taken at various focus level. 